\title{
DEUTSCHE
}

\section{MEDICINISCHE WOCHENSCHRIFT.}

\section{Mit Berücksichtigung des deutschen Medicinalwesens nach amtlichen Nittheilungen, der iffent- lichen Gesundheitspflege und der Interessen des ärztlichen Standes.}

\author{
Begrïndet von Dr. Paul Börner,
}

\author{
Zwanzigster Jahrgang.
}

\author{
Redaction: Prof. Dr. A. Eulenburg und Dr. Jul. Schwalbe, Bcrin. - Verlag: Georg Thieme, Leipzig-Berlin. \\ Lichtensteinallee 3.

\section{Aus der Kinderklinik der Königlichen Charité. Ueber larvirte Diphtherie. Von 0. Henbner.}

In der gegenwärtigen Zeit, wo der Kliniker mit besonders gespannter Aufmerksamkeit den Ablauf der Erscheinungen in jedem Fall von Diphtherie verfolgt, dürfte es nicht werthlos sein, daranf aufmerksam zu machen, dass es Fälle von Diphtherie giebt, welche auch dem aufmerksamen Beobachter während eines Theils ihres Verlaufes, ja selbst bis zum Tode verborgen bleiben.

Dem Familienarzt werden solche Fälle selten in den Gesichtskreis kommen. Ich selbst habe vor Jahren zwei Fälle beschrieben, die in dieses Gebiet gerechnet werden könnten 1 ). Dem Hospitalarzt kommen sie wohl häufiger vor. Schon im Leipziger Kinderkrankenhause machte ich hierher gehörige Beobachtungen. Aber erst das Nachdenken über einige hier vorgekommene Erkrankungen mit ungewöhnlichem Verlaufe verdichtete die bisherigen Vorstellungen zu einem etwas deutlicheren Bilde.

Es handelt sich immer um secundäre Diphtherieen in dem Sinne, wie wir von secundären Masern, secundärem Scharlach und dergleichen sprechen. Die Infection befüllt nicht gesunde, sondern bereits vorher kranke oder kränkliche Kinder, welche einen länweren Aufenthalt im Krankenhause hinter sich haben. Dabei wird das Contagium durch Besucher oder auch auf irgend eine andere Weise eingeschleppt, die später gewöhnlich nicht mehr klarzusteller ist. Nun aber erkrankt das Kind nicht in der gewöhnlichen Weise mit entzündlichen Ausschwitzungen in den Rachentheilen, mit heftigem Fieber, Erbrechen, Drüsenschwellungen u. s. w. sondern mit zunächst wenig alarmirenden, katarrhalischen Erscheillungen sei es der Athmungs-, sei es der Verdauungsorgane, wenig charakteristischem Fieber und nicht auffallend von dem früheren Zustande sich abhebender Verschlimmerung des Allgemeinbefindens. Man findet die Beeinträchtigung des Befindens genügend durch die nachweisbaren katarrhalischen Erscheinungen, z. B. auf der Linge, erklärt und ahnt nicht, dass eine tödtliche Erkrankung heimtückisch zum letzten entscheidenden Angriff auf den Organismus sich vorbereitet - bis auf einmal, scheinbar ganz plötzlich, das Auftreten einer schweren Kehlkopfstenose auf die vorher latente Gefahr in erschreckender Weise aufmerksam macht. Ja, selbst an der Leiche kann es sich erst aufklären, dass die: Todesursache, wenn nicht allein, so doch zum. Theil durch eine diphtherische Infection mit bedingt gewesen war.

Zur Erläuterung dieses larvirten oder latenten Verlaufes der Diphtherie seien folgende Beobachtungen angeführt.

Fall 1. L., Hedwig, 11/2 Jahre alt, wird am 27. April 1894 wegen schwerer Rachitis und eines noch nicht verheilten, seit 16 Tagen bestehenden Oberschenkelbruches aufgenommen. Weit offere Fontanelle, Kyphose der Lendenwirbel, Verdickung der Knorpelknochengrenze der Rippen, Verkrummung der Beine. Sehr langsame Consolidirung des fracturirten Knochens. Grobe Bronchitis. Während des Mai ab und zu kleine Fiebersteigerungen, die auf die Bronchitis bezogen wurden.

Am 23. Mai stieg die Temperatur auf 38,1 , am 24. auf 39,1 , und nun schloss sich bis zum 1. Juni eim geringes remittirendes Fieber an, mit höchster Erhebung auf 38,8. Dasselbe schien abhängig von einem ziemlich intensiven Schnupfen, zu dem sich stärkerer Husten und Zeichen einer mässig ausgebreiteten Katarrhalpneumonie im rechten Unterlappen gesellten. Die Rachenorgane zeigten durchaus keine Abweichungen von der Norm. Vom 3. bis 5. Juni war das Kind wieder fieberlos, hustete aber fort.

\footnotetext{
I) Jahrb. f. Kinderheilkunde VI, S. 105.
}

Am 6. Juni hob sich aber das Fieber wieder auf 38,0 Abends. Das Kind war etwas heiser und hatte Nachts stark geschwitzt.

Am 7 Juni nimmt bei gleichem geringem Fieber die Heiserkeit.

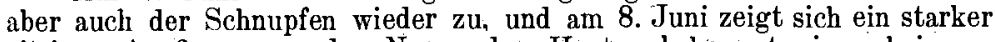
eitriger Ausfluss aus der Nase; der Husten bekommt einen heiseren Klang. Das Fieber erhebt sich weiter auf 39,3, Puls 168.

Am 9. Juni trüh fällt zum erstenmal ein ganz leichtes Stenosengeräusch auf und eine vermehrte Athemfrequenz von 40 Respirationen. Jetzt kam mir die Erinnerung an einen ähnlichen in Leipzig beobachteten Fall, und obgleich im Rachen nichts Auffälliges zu sehen war, obwohl der Appetit leidlich und der Stuhl normal waren, ordnete ich sofort die Verlegung des Kindes nach der Beobachtungsstation an.

Die Befurchtung war nur zu - begründet, denn bereits Nachmittags 4 Uhr sind, unter Steigerung der Temperatur auf 40,2, deutliche Zeichen der Larynxstenose vorhanden. welche die Intubirung nöthig machen. Gleichzeitig wird eine Dosis Heilserum (von dem Institut fur Infectionskrankheiten gutigst überlassen) injicirt. $\mathrm{Zu}$ spät. Die Athemnoth wird nicht geringer, der Puls immer kleiner; auch die Nachts $11^{\mathrm{I}} / 2$ Uhr noch vorgenommene Tracheotomie bessert die Lage nicht: die hohe Athemnoth und Pulsfrequenz dauern an, während die Temperatur auf 40,8 steigt, und am 10. Juni, Vormittags $11^{\mathrm{I}} / \mathbf{2}$ Uhr, geht die Kleine in tiefem Collaps zugrunde.

Die Autopsie (Dr. Hansemann ${ }^{1}$ ) ergiebt folgendes: Ziemlich gut genahrtes Kind weiblichen Geschlechts mit starker Rachitis am Thorax und den Extremitätenknorpeln. Der rechte Oberschenkel zeigt eine mit weichem Callus versehene Fractur. Tracheotomiewinde.

Rachen-, Nasen- und Highmorshöhle zeigen leicht geröthete, aber sonst unveränderte Schleimhaut. Tonsillen nicht geschwollen. - Vom unteren Rande der Epiglottis bis einige Millimeter unter den Stimmbändern zeigt die Schleimhaut weisslich gelbe ziemlich trockene- Einlagerungen, die sich nicht leicht abziehen lassen und zum Theil bis in die Submucosa reichen. Durch dieselbe ist der Eingang zum Kehlkopf fast vollständig verschlossen. Weitere geschwurige Defecte oder ältere Schorfe sind nicht vorhanden. Unterhalb dieser Partie ist die Schleimhaut der Trachea leicht geröthet und mit einer schmierig truben. Flussigkeit bedeckt.: Die Consistenz der Lungen ist etwas vermehrt, dieselben sind aber im allgemeinen lufthaltig.

Herz ist blass, sonst ohne Besonderheiten. Milz etwas vergrössert, Follikel deutlich. Nieren blass, ohne Besonderheiten, ebenso Leber. Darmschlingen meteoristisch aufgetrieben.

Es kann keinem Zweifel unterliegen, dass hier die Diphtherie nicht etwa erst an dem Tage der ersten Stenosenerscheinungen eingesetzt hat. Die vom 6 . Juni an sich steigernde Heiserkeit weist ebenso wie das begleitende Fieber deutlich genug darauf hin, dass die Infection des Larynx mindestens vier Tage vor den letalen Ausgang zurückzudatiren war. - Ja noch mehr, ich halte es für sehr wahrscheinlich, dass bereits die ganze, am 23. Mai begonnene Schnupfenkrankheit auf eine diphtherische Infection zurückzubeziehen war. Allerdings fehlt hier der bacteriologische Beweis; man war eben bei der Geringfügigkeit der Erscheinungen, dem fortdauernd guten Appetit, dem wieder zurückgehenden Fieber nicht auf den Gedanken gekommen, dass eine Diphtherie vorliegen könne.

1) Herr Dr. Hansemann hat in seinem Vortrag in der medicinischen Gesellschaft zu Berlin aber Diphtherie (Berliner klinische Wochenschrift No. 50) eine Beobachtung aus meiner Klinik veröffentlicht, für deren Darstellung ich keine Verantwortung trage. Sie ist vielleicht identisch mit der obigen. Genau kann ich es nicht sagen, da Herr Hansemann-mir über seine Absicht, einen meiner Fälle zu benutzen, eine Mittheilung nicht hat zukommen lassen. Ich bedauere die Unterlassung dieser sonst wohl allgemein üblichen Hoflichkeit deshalb, weil ich vielleicht in der Lage gewesen wäre, ihm zu einer etwas exacteren Krankengeschichte 'zu' verhelfen, als' er seinen Zuhörern geboten ' hat: 
Wohl möglich, dass der Verlauf auch in der folgenden Beobachtung sich ähnlich gestaltet hätte, wenn wir nicht, gewitzigt durch die soeben mitgetheilte Erfahrung, da sofort eingegriffen hätten.

Fall 2. M., Amelie, 13 Monate alt, eine Bettnachbarin des vorigen Kindes, war am 30. Mai wegen starker Anämie und hochgradiger Rachitis in die Kinderabtheilung aufgenommen worden. Fin anfängliches durch Verdauungsstörung bedingtes. Fieber hatto sich rasch gehoben, und es ging der Kleinen ganz leidlich, als sie plotzlich am 12. Juni bei fieberlosem Zustand (37.4) eine heftige Rhinitis bekam.

Sofort wurde die Kleine auf die Beobachtungsstation verlegt und bekam am selben Abend, noch ehe die bacteriologische Diagnose gestellt werden konnte, eine Dosis Heilserum injicirt. Denn auch die Rachenorgane zeigten sich etwas gerothet und geschwollen, an den Tonsillen eine ganz leichte graue Verfärbung. Submaxillardrijsen etwas geschwollen. A uf den Lungen einige katarrhalische Geräusche.

A m 13. J uni steigt das Fieber auf 39.2, sinkt aber dann rasch auf 37.7, um sich am 14. November auf 38,5 zu heben und dann definitiv in Fieberlosigkeit tberzugehen. Schnupfen und Rachenaffection gehen rasch zurück. Am Körper erscheint ein Urticariaexanthem. welches am 15. Juni wieder zurückgeht. Die aus dem Nasenschleim angelegten Culturen erweisen sich als charakteristische Diphtheriebacillen.

Von 27 . Juni bis 1. Juli fiebert das Kind nochmals wegen einer Verdauungsstörung. Dann Besserung. Am 4. Juli wird das Kind in gutem Zustande entlassen.

Die beschriebenen beiden Fälle wurden die Veranlassung, dass bei sämmtlichen kleinen Mitbewohnern der Abtheilung eine Immunisirungseinspritzung von Heilserum vorgenommen wurde. Es kam unter diesen kein weiterer Erkrankungsfall vor.

Erst ganz neuerdings hatten wir wieder eine Infection zu beklagen, welche aber klinisch vollständig latent verlief.

Fall 3. Sch., Otto. 2 Jahre, wurde am 3. November d. J. aufgenommen. Er ist immer schwächlich und bleich gewesen. Der Grossvater starb an Lungenschwindsucht. Hat von jeher trocken gehustet und wurde leicht kurzathmig. Seit drei Wochen hat er dünne schleimige Stithle, seit 14 Tagen magert er ab; hat angeblich ab und zu Fieber. Am rechten Ohre hatte er ofters etwas Ausschlag, seit acht Tagen sind die rechtsseitigen Halsdrüsen geschwollen.

Bei der Aufnahme findet man ein dürftig entwickeltes, blasses, graciles Kind, linksseitige Trubung der Hornhaut, beiderseits scrofulöse Drüsenpackete. An den inneren Organen nichts krankhaftes nachweisbar.

Der Knabe wird mit Leberthran. guter Ermährung und vorsichtigen Tuberkulindosen behandelt. die von Reaction nicht gefolgt sind. Vom 3. bis 19. November absolute Fieberlosigkeit und gutes Befinden. Am 20. November erhebt sich das Fieber plotzlich auf 39.2. sinkt in der Nacht auf 36.9. um aber am 21. und 22. November auf 40 und 40,3 sich zu erheben. jeden Morgen geht die Temperatur auf 37 zurtick; am 23. November höchste Temperatur 38,3 , am 24 . November 39,0 , am 25 . November wieder Abfall auf 38.2

1)ie Erscheinungen, die der Knabe sonst darbot, bestanden in Appetitverminderung und geringem feinem Rasseln über der dritten und vierten Rippe an der rechten Vorderwand des Thorax; am 23. November gesell to sich heftiges Erbrechen, das sich in den folgenden Tagen wiederholte, Verfall und Kuhlwerden hinzu. Am 24. November grosse Apathie, viel Schlaf, ab und zu trockner Husten, dünne schleimige Ausleerungen. Am 25. November vollige Apathie, immer leichter Schlummer. Puls sehr klein, frequent, fadenforrmig. Erbrechen nach jeder Nahrungsaufnahme, sehr zahlreiche. schleimige, stark stinkende Stuhle. Vormittags $1 / 212 \mathrm{Uhr}$ Tod in collabirtem Zustand.

Sectionsprotokoll (Dr. Hansemann): Im Pharynx, auf den Tonsillen, auf der Epiglottis bis auf die Stimmbänder herab flache grauweisse Auflagerungen; die sich nur unter Verlust der Schleimhaut abziehen lassen. weiter in der Trachea zarte fibrinose Auflagerungen auf der gerötheten Schleimhaut. DieLymphdrúsen am Halse markig geschwollen, ebenso diejenigen um die Bronchien herum. Eine grossere und eine kleinere von den letzteren sind verkäst, von gelber, ziemlich trockener Beschaffenheit. Tuberkel finden sich nirgends. Die Schleimhant der Bronchien ist bis in die feinsten Verzweigungen hinein gerothet und mit feinen fibrinösen Auflagerungen bedeckt.

In der ganzen Lunge zahlreiche frische bronchopneumonische Heerde. Die Pleuren beiderseits verwachsen durch zarte fibröse Stränge. Die Mesenterialdrtisen sind markig geschwollen, frei von jeder Verkäsung und von Tuberkeln.

Milz ist nicht vergrössert. Nieren ohne Besonderheit. Die Magenschleimhaut stark geröthet.

Gross war die Verwunderung angesichts dieses Leichenbefundes. Dass es sich um echte Diphtherie handelte, erwies die bacteriologische Untersuchung der dünnen Auflagerung in der Trachea, aus welcher typische Diphtheriebacillenculturen aufgingen. -- Und doch war kein Zug in dem klinischen Krankheitsbilde, welcher auch nur entfernt an eine lokale Erkrankung der Rachen- oder Kehlkopfschleimhaut hätte denken lassen, kein Schmerz, keine' Schlingbeschwerden, keine Andeutung von Kehlkopfstenose, Heiserkeit und dergleichen.

Die Allgemeinerscheinungen deuteten freilich auf eine schwere Infection hin, indessen wäre man nach den Erscheinungen während des Lebens viel eher auf eine schwere Magendarmstörung gefasst, als auf Diphtherie. Der Magen war ja in der That stark afficirt, über den Darm findet sich keine Angabe. - Es kann aber wohl nicht bezweifelt werden, dass die diphtherische Infection hier dasjenige wesentliche Leiden war, welches dem gesamtimten schweren Symptomenbilde der letzten sechs Luebenstage zugrunde lag.

Fragt man sich gegenüber solchen Fällen wie die beschriebenen, auf welche Weise so betrachtliche Abweichungen des klinischen Bildes einer sonst in ziemlich regulärem Typus ablaufenden Krankheit beding* sein kónnen, so scheinen mir hauptsächlich zwei Punkte in Betracht zu kommen.

Vor allem der betroffene Organismus. Es sind schwächliche vou constitutionellen Lueiden (Rachitis, Scrofulose) heimgesuchte und sodann noch sehr junge Organismen, die befallen werden. Es ist ja auch bei sonst gesunden sehr jungen. Kindern nicht selten, dass z. B. die Rachenerscheinungen bei der Diphtherie wenig vortreten und deshalb die Krankheit oft gleich im Kehlkopf zu beginnen scheint, und handelt es sich dann noch um kranke und sehr junge Organismen, so wird es begreiflich, dass die Gesammtreaction gegen die Infection eine viel schwächere ist und dadurch ein so abgeblasstes oder selbst verändertes klinisches Bild entsteht. - Was namentlich die schweren Erscheinungen seitens des Digestionstractus in dritten Falle anlangt, so wissen wir ja aus den Versuchen von Roux und Yersin, dass bei Infectionen mit dem reinen Diphtherietoxin häufig Diarrhöen bei Thieren auftreten; - es drängte sich also eine sonst beim Menschen nebensächliche oder fehlende Erscheinung bei unserem Falle in den Vordergrund, während die klinischen Haupterscheinungen der gewöhnlichen Fälle hier vollständig latent blieben.

Ein zweiter Umistand, welcher geeignet ist, die Diagnose in solchen Fällen zu erschweren, liegt darin, dass die an sich schon blassen und verschwommenen Zige des Krankheitsbildes nicht einmal irgend einen besonderen Charakter tragen. Vielmehr sind sie ganz wohl mit dem Grundleiden, wegen dessen das Kind aufgenommen wurde, in Einklang zu bringen. So waren z. B. in Fall 1 ja schon während des ersten Monats des Krankenhausaufenthaltes mehrfach kleine Fiebersteigerungen mit Exacerbationen der rachitischen Bronchitis aufgetreten. Warum sollte man bei dem Auftreten des fieberhaften Schnupfens an Fernliegendes denken und nicht vielmehr an eine acute Verschlimmerung des chronischen Katarrhs der Respirationsorgane? - Ebenso hatte das Kind 3 schon öfter's an Verdauungsstörungen gelitten, warum sollte man sich nicht zunächst bemühen, dieser Verdauungsstörung wieder Herr zu werden, in der Hoffnung, damit auch den Fieberzustand wieder zu beseitigen? So trägt gleichsam die primäre chronische Krankheit selbst dazu bei, den neuen, secundär hinzugetretenen Feind dem Auge des Arztes zu verhüllen.

In der Litteratur ist dieser Kategorie von Erkrankungen noch nicht viel Beachtung geschenkt worden. Jacobi kennt sie offenbar, wenn er die geringfügigen Erscheinungen, welche dem plötzlichen Auftreten der Kehlkopfstenose bei seiner "primären Diphtherie der Trachea" vorhergehen, schildert.1) Er "sagt aber nicht, dass es sich dabei meist um secundär $\theta$ Diphtherieen (im obigen Sinne) handelt.

Eine Lehre möchte man vielleicht aus den dargestellten Beobachtungen ziehen: überall, wo plötzliche Veränderungen im Befinden eines constitutionell kranken Kindes auftreten, die mit Fieber und mit katarrhalischen Erscheinungen verbunden sind, die bacteriologische Diagnostik zu Hülfe zu nehmen. Dass man, wenı man früh genug zur Diagnose gelangt, auch in solchen Fällen vielleicht zu helfen vermag, zu dieser Hoffnung scheint die zweite Beobachtung zu ermuthigen. 